This document is the accepted manuscript version of a published work that appeared in final form in Nature Nanotechnology after peer review and technical editing by the publisher. To access the final edited and published work see https://www.doi.org/10.1038/s41565-019-0594-8

\title{
Reversible coordination-induced spin-state switching in complexes on metal surfaces
}

\author{
Alexander Köbke, ${ }^{1}$ Florian Gutzeit, ${ }^{2}$ Fynn Röhricht, ${ }^{2}$ Alexander Schlimm, ${ }^{3}$ Jan Grunwald, ${ }^{3}$ Felix Tuczek, ${ }^{3}$ \\ Michał Studniarek, ${ }^{4}$ Danilo Longo, ${ }^{5}$ Fadi Choueikani, ${ }^{5}$ Edwige Otero, ${ }^{5}$ Philippe Ohresser, ${ }^{5}$ Sebastian \\ Rohlf, ${ }^{1,6}$ Sven Johannsen, ${ }^{1}$ Florian Diekmann, ${ }^{1,6}$ Kai Rossnagel, ${ }^{1,6,7}$ Alexander Weismann, ${ }^{1}$ Torben \\ Jasper-Toennies, ${ }^{1}$ Christian Näther, ${ }^{3}$ Rainer Herges* ${ }^{2}$ Richard Berndt, ${ }^{1}$ and Manuel Gruber ${ }^{1, *}$ \\ ${ }^{1}$ Institut für Experimentelle und Angewandte Physik, Christian-Albrechts-Universität zu Kiel, 24098 Kiel, Germany \\ ${ }^{2}$ Otto-Diels-Institut für Organische Chemie, Christian-Albrechts-Universität zu Kiel, 24098 Kiel, Germany \\ ${ }^{3}$ Institut für Anorganische Chemie, Christian-Albrechts-Universität zu Kiel, 24118 Kiel, Germany \\ ${ }^{4}$ Swiss Light Source, Paul Scherrer Institute, 5232 Villigen PSI, Switzerland \\ ${ }^{5}$ Synchrotron SOLEIL, L'Orme des Merisiers, Saint-Aubin - BP 48, 91192 Gif-sur-Yvette, France \\ ${ }^{6}$ Ruprecht-Haensel-Labor, Christian-Albrechts-Universität zu Kiel und Deutsches \\ Elektronen-Synchrotron DESY, 24098 Kiel und 22607 Hamburg, Germany \\ ${ }^{7}$ Deutsches Elektronen-Synchrotron DESY, 22607 Hamburg, Germany
}

Molecular spin switches are attractive candidates for controlling the spin polarization developing at the interface between molecules and magnetic metal surfaces ${ }^{1,2}$, which is relevant for molecular spintronics devices ${ }^{3-5}$. However, intrinsic spin switches such as spin-crossover complexes so far suffer from fragmentation or loss of functionality upon adsorption on metal surfaces with rare exceptions ${ }^{6-9}$. Robust metal-organic platforms, on the other hand, rely on external axial ligands to induce spin switching ${ }^{10-14}$. Here we integrate a spin switching functionality into robust complexes relying on the mechanical movement of an axial ligand strapped to the porphyrin ring. Reversible interlocked switching of spin and coordination, induced by electron injection, is demonstrated for this class of compounds on $\mathrm{Ag}(111)$. The stability of the two spin and coordination states of the molecules exceeds days at $4 \mathrm{~K}$. The potential applications of this switching concept goes beyond spin and may turn out useful for controlling the catalytic activity of surfaces ${ }^{15}$.

The control of axial ligand coordination to a Ni porphyrin, and henceforth control of the Ni-center spin, may be achieved by an integrated molecular switch such as an azopyridine moiety ${ }^{16}$. However, this function is lost on metal surfaces ${ }^{17}$ because the moleculesubstrate interaction modifies the flexible azopyridine moiety. We developed an alternative strategy using strapped porphyrins ${ }^{15,18,19}$ with a pyridine moiety in the strap for axial coordination (Fig. 1a). In contrast to previously synthesized strapped porphyrins, we designed rigid straps to ensure surface compatibility and to mechanically couple the pyridine moiety to the conformation of the porphyrin platform (flat/ruffled), which in turn is coupled to the $\mathrm{Ni}$ spin state.

$\mathrm{Ni}$ porphyrins may be optically switched from a lowspin (LS) $S=0$ ground state to a high-spin (HS) $S=1$ state with a short lifetime on the order of $200 \mathrm{ps}^{20}$. The goal of our molecular design is to stabilize these spin states by an intramolecular feedback mechanism: The Ni cation in the HS (LS) state favors a flat (ruffled) shape of the porphyrin ${ }^{21}$. The flat (ruffled) shape of the porphyrin stretches (compresses) the strap as to keep the pyridine ligand coordinated (non-coordinated). In turn, the coordination (non-coordination) of the axial ligand stabilizes the HS (LS) state (Fig. 1a). ${ }^{22}$
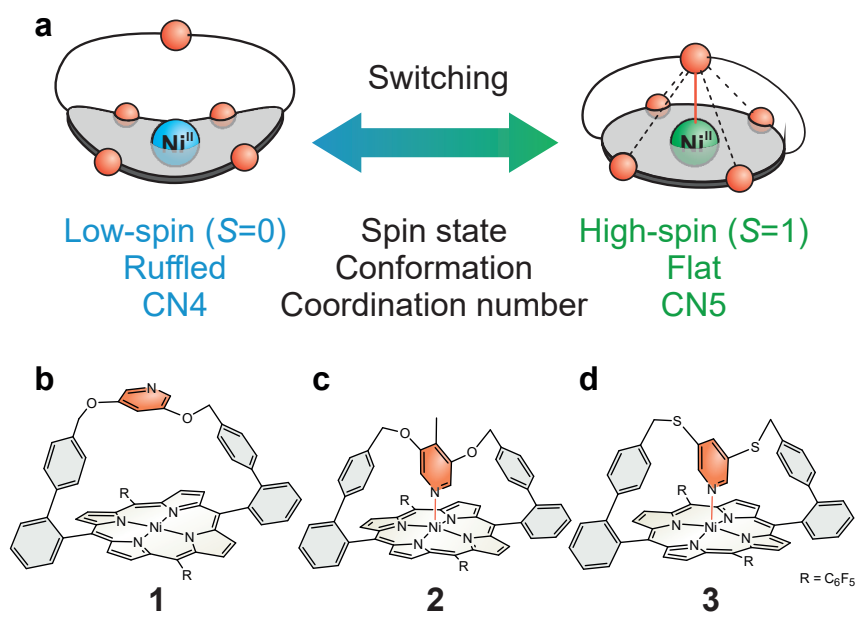

FIG. 1. Molecular switch with interlocked coordination and spin degrees of freedom. a In the LS state (left) the pyridin moiety is decoordinated, the complex is square planar (CN4). In the HS state (right) the pyridin coordinates as the axial ligand of the square pyramidal (CN5) complex. b-d We synthesized complexes $\mathbf{1}, \mathbf{2}$ and $\mathbf{3}$ implementing this concept. They differ by a constituent of the strap $(\mathrm{X}=\mathrm{O}, \mathrm{S})$ or by pyridine substitution, determining the molecular ground state (CN5 or CN4). The molecular models are based on $\mathrm{x}-$ ray crystallographic data acquired at ambient temperature (SI section XII).

The length and rigidity of the strap and the binding affinity of the pyridine ligand determine whether the LS or the HS state is the ground state of the compound. We synthesized three compounds, namely 5,15-(2'-(4"'(3"',5"'-pyridinylen)-oxymethylphenyl)-phenyl)-10,20bis-(2,3,4,5,6-pentafluorophenyl)-Ni(II)porphyrin $\quad \mathbf{1}$, 
5,15-(2'-(4"'-(4"' -methylpyridin-3"' ,5"' '-ylen)-oxymethylphenyl)-phenyl)-10,20-bis-(2,3,4,5,6-pentafluorophenyl)$\mathrm{Ni}(\mathrm{II})$ porphyrin 2, and 5,15-(2'-(4"'-(3"', ,5"'-pyridinylen)sulphonylmethylphenyl)-phenyl)-10,20-bis $(2,3,4,5,6$ pentafluorophenyl)-Ni(II)porphyrin $\mathbf{3}$, respectively shown in Figs. $1 \mathrm{~b}-\mathrm{d}$, with varying constituents in the strap (O or $\mathrm{S}$ ) and with or without a methyl group on the pyridine ligand. These compounds have different properties at ambient temperature in solution (Supplementary Information, SI section I). A dynamic equilibrium is observed where the fractions of molecules in the coordination number $(\mathrm{CN}) 5$ state, i.e., with the axial ligand coordinated to the Ni cation, are $18 \%$ (1), $57 \%$ (2) and $100 \%(3)$. This shows that the energy difference between the LS and the HS states is on the order of the thermal energy for $\mathbf{1}$ and $\mathbf{2}$. The coordinations found in solution are also present in the crystalline state (SI sections I and XII). The derivative $\mathbf{1}$ is in the CN4 LS state, i.e., with the axial ligand not coordinated, while compound $\mathbf{3}$ is in the CN5 HS state $^{23}$. In contrast, the $\mathrm{CN}$ of $\mathbf{2}$ in the crystalline state depends on the crystallization conditions and can either be CN4 or CN5 (SI section XII). This, along with the dynamic equilibrium observed in solution, is evidence that the HS and LS states of $\mathbf{2}$ are close in energy and the ground state depends on the environment.

To further investigate the interplay between structural and electronic/magnetic properties, we employed nearedge $\mathrm{x}$-ray absorption fine structure (NEXAFS) along with x-ray magnetic circular dichroism (XMCD). As a reference 5,15-bis(2,3,4,5,6-pentafluorophenyl)-10,20bisphenyl-Ni(II)porphyrin4 was used, which contains no pyridine moiety and has an expected spin $S=0$ (SI section II). Figures $2 \mathrm{a}-\mathrm{b}$ show the $\mathrm{x}$-ray absorption (XA) and the XMCD spectra at the $\mathrm{Ni} L_{2,3}$ edges acquired on powder samples of complexes $\mathbf{1}-\mathbf{4}$. The XA spectra of $\mathbf{4}$ and $\mathbf{1}$ (violet and blue curves in Fig. 2a) are very similar to data from $\mathrm{Ni}^{2+}$ porphyrin complexes in the LS state ${ }^{24}$. The corresponding XMCD spectra show no or little dichroism (violet and blue curves in Fig. 2b), as expected for $S=0$. The small dichroism of $\mathbf{2}$ may actually be due to a small fraction of complexes in the coordinated HS state. In contrast, the XA spectra of $\mathbf{2}$ and $\mathbf{3}$ are significantly different and match those of HS $\mathrm{Ni}^{2+}$, while their magnetic dichroism is compatible with $S=1$ (red and green curves in Figs. 2a - b). From the NEXAFS and XMCD data we conclude that, in powder, complexes $\mathbf{2}$ and $\mathbf{3}$ are in a CN5 HS $(S=1)$ state, while 4 and 1 are in an CN4 LS $(S=0)$ state. These results are in agreement with crystallographic data (SI section XII). Furthermore, we performed multiplet calculations using the positions and charges of the $\mathrm{N}$ atoms as inferred from gas-phase density functional theory calculations. A satisfactory agreement is found between the simulated multiplets and the XA spectra (SI section V) confirming the interplay between structural and spin states.
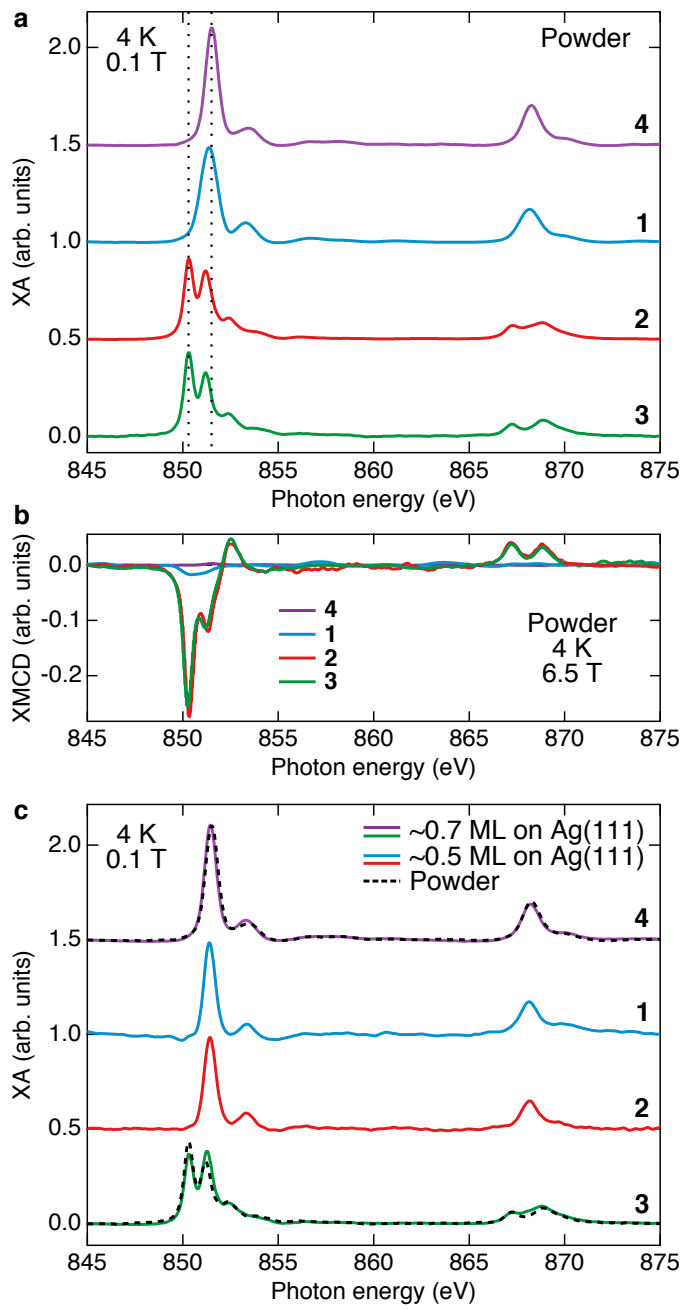

FIG. 2. Interlocking of coordination and spin states. a NEXAFS spectra at the $\mathrm{Ni} L_{3,2}$ edges acquired on powder samples of complexes 1-3 (Fig. 1b) and of a Ni-porphyrin reference 4 . The CN4 (CN5) complexes 4 and $\mathbf{1}$ (2 and $\mathbf{3}$ ) exhibit spectra characteristic of the $\mathrm{Ni}^{2+} \mathrm{LS}(\mathrm{HS})$ state. The dotted lines are guide to the eye. $\mathbf{b}$ XMCD performed at $4 \mathrm{~K}$ under a magnetic field of $6.5 \mathrm{~T}$ of the different powders. The strong dichroism observed for compounds $\mathbf{2}$ and $\mathbf{3}$ evidences that these compounds are magnetic. c The successful deposition and the absence of significant change in the oxidation state of the molecular complexes upon adsorption on $\operatorname{Ag}(111)$ is evident from the similar NEXAFS spectra of $\approx 0.7$ monolayer $\mathbf{4}$ and $\mathbf{3}$ and $\approx 0.5$ monolayer $\mathbf{1}$ and $\mathbf{2}$ on $\mathrm{Ag}(111)$ (colored lines) to the reference spectra acquired on the respective powders (dashed black lines). For complex $\mathbf{2}$, the ground state changes from HS CN5 in the powder form to LS CN4 for the compound adsorbed on $\mathrm{Ag}(111)$. ML stands for monolayer.

In a next step, we verified the intactness of the compounds and their function upon in vacuo sublimation on $\mathrm{Ag}(111)$. XA spectra of sub-monolayer coverage of $\mathbf{4}$, $\mathbf{1}$ and $\mathbf{3}$ on $\mathrm{Ag}(111)$ (violet, blue and green curves in Fig. 2c) are very similar to the XA spectra of the respective powders (dashed black curves in Fig. 2c). This 
is evidence that the oxidation state and the ligand field of the Ni cation are essentially unaffected by adsorption on the $\operatorname{Ag}(111)$ surface, because such changes would lead to a modification of the XA spectra. Consequently, the magnetic properties of compounds $\mathbf{1}, \mathbf{3}$ and $\mathbf{4}$ are robust against adsorption on $\mathrm{Ag}(111)$ as confirmed by XMCD measurements (SI section IV). Furthermore, the similarities between the spectra acquired on powders and adsorbed molecules indicate a limited hybridization between the $\mathrm{Ni}$ cation and the substrate. In the LS state, the $d_{z^{2}}$ orbitals are filled, while in the HS state, the flat conformation of the macrocycle along with the perpendicular orientation of surrounding phenyl rings ensure a sufficiently large Ni-Ag(111) distance. Both effects limit the hybridization of the $\mathrm{Ni}$ to the substrate. For $\mathbf{2}$, the ground state of the compound changes from HS in the powder form to LS on the $\mathrm{Ag}(111)$ surface (Figure 2a,c), emphasizing again the bistability of this complex. For completeness, we note that additional axial coordination with $\mathrm{Ag}$ atoms is excluded because the spectra acquired on complexes 1, 2 and $\mathbf{4}$ adsorbed on $\mathrm{Ag}(111)$ are characteristic of CN4 Ni complexes (SI section V).

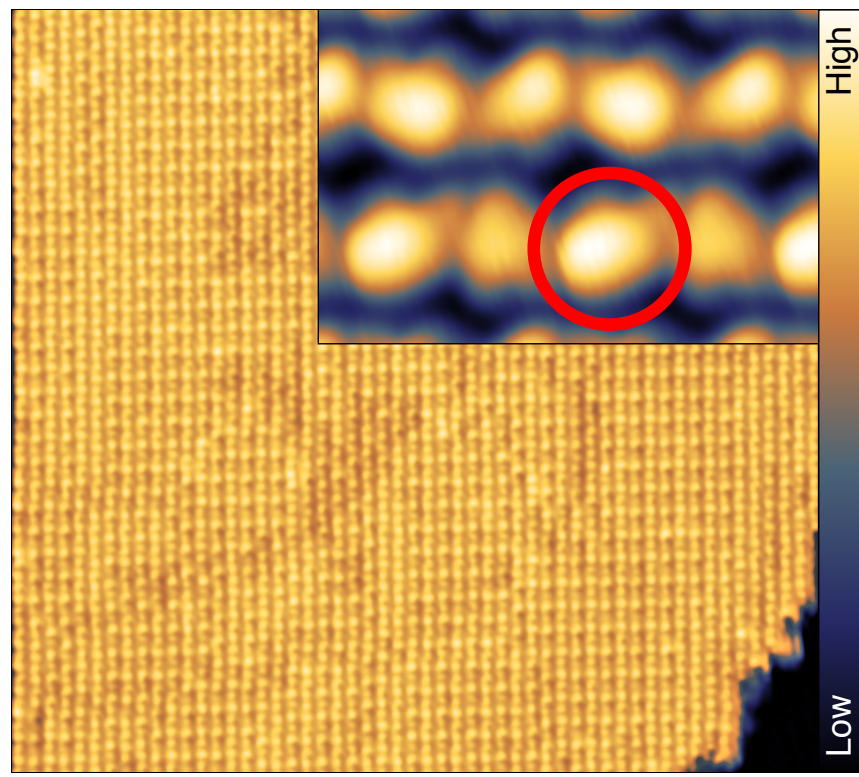

FIG. 3. Sub-monolayer coverage of 2 on $\operatorname{Ag}(111)$. Constant-current STM topograph of $\mathbf{2}$ on $\mathrm{Ag}(111)$ (1 V, $3 \mathrm{pA}$, $90 \mathrm{~nm}$ wide) exhibiting a wide molecular island (yellowish color). The inset ( $1 \mathrm{~V}, 10 \mathrm{pA}, 6 \mathrm{~nm}$ wide) shows that the individual complexes 2 (bright protrusions, example surrounded by a red circle) have different apparent heights (up to $80 \mathrm{pm}$ variation) presumably because of intermolecular interactions. The color scale shown on the right is used throughout the manuscript. Here it spans over $610 \mathrm{pm}$ ( $450 \mathrm{pm}$ for the inset).

To probe the switching properties of the molecules, we employed scanning tunneling microscopy (STM) at $4 \mathrm{~K}$. For this purpose, compound $\mathbf{2}$ is the most interesting candidate because the data from solution and solid samples suggest that the energy difference between its spin states is the smallest of all investigated compounds. Fig. 3 shows a topograph of a $90 \mathrm{~nm}$ wide island of $\mathbf{2}$ on $\mathrm{Ag}(111)$. The molecules most probably adsorb flat with the porphyrin on the metal, as observed for other porphyrin complexes on metals ${ }^{17,25,26}$, with the strap pointing away from the surface.

Switching of individual molecules is achieved by increasing the sample voltage $V$ to $2.1-2.7 \mathrm{~V}$ for a few seconds (Figs. 4a - c, see Extended Data Fig. 1) and leads to an apparent-height increase of $\approx 100-150 \mathrm{pm}$ in addition to the initial corrugation of the molecule. As discussed below, this change corresponds to a transition from the CN4 LS $(S=0)$ to the CN5 HS $(S=1)$ state. The molecular state may be converted back by applying $V \approx-1.8 \mathrm{~V}$ (Extended Data Fig. 1). In total, hundreds of molecules were successfully and reversibly switched (Extended Data Figs. 1 and 2). We never observed spontaneous back switching, even after days of measurements.

The identification of the molecular states relies on both the energy change of the molecular orbitals upon switching in comparison to reference systems and on the presence/absence of a zero-bias feature in the differentialconductance spectra. The differential conductance spectrum acquired atop a complex $\mathbf{2}$ in its pristine LS state exhibits a peak at $-2.4 \mathrm{~V}$ (upper blue curve in Fig. $4 \mathrm{~d}$ ), corresponding to the highest-occupied molecular orbital (HOMO). The HOMO energy is essentially independent of the apparent height of the pristine complexes (SI section VII). In the HS state, the HOMO is shifted upward by $\approx 0.55 \mathrm{eV}$ (upper dashed green curve in Fig. $4 \mathrm{~d}$ ). This effectively leads to a reduced HOMO-LUMO gap for the HS state, in agreement with gas-phase density functional theory calculations (SI section III). The $d I / d V$ spectrum acquired on the molecule switched back to the LS state is virtually identical to that of the pristine LS molecule.

To independently test the spin state dependence of the HOMO energy, we used $d I / d V$ spectra of $\mathbf{3}$ and $\mathbf{1}$. As the HOMO is predominantly localized on the porphyrin ligand (SI section III), only minor shifts of the HOMO between the different compounds are expected. The HOMO energy of $\mathbf{1}$ on $\mathrm{Ag}(111)$ in its pristine LS state is $\approx-2.3 \mathrm{eV}$ (middle blue curve in Fig. $4 \mathrm{~d}$ ), i.e., comparable to that of $\mathbf{2}$ in the LS state $(-2.4 \mathrm{eV})$. Similarly to $\mathbf{2}$ on $\operatorname{Ag}(111)$, reversible switching of $\mathbf{1}$ between the CN4 LS and the CN5 HS states was realized by STM (SI section IX) and is accompanied by a similar HOMO shift $(0.55 \mathrm{eV})$. The HOMO energies of both HS 1 and HS $\mathbf{2}$ complexes are again comparable (Fig. 4d) and confirm the spin state assignment. A further comparison is made with the HOMO of HS 3 on $\mathrm{Ag}(111)$, which is in the CN5 HS state as evident from the NEXAFS data (Fig. 2c). The HOMO energy $(\approx-1.8 \mathrm{~V})$ matches that of the other HS complexes (Fig. 4d). Therefore, compounds 1-3 have similar state-dependent HOMO energies, which allows to identify the state of a molecule through the energy of 

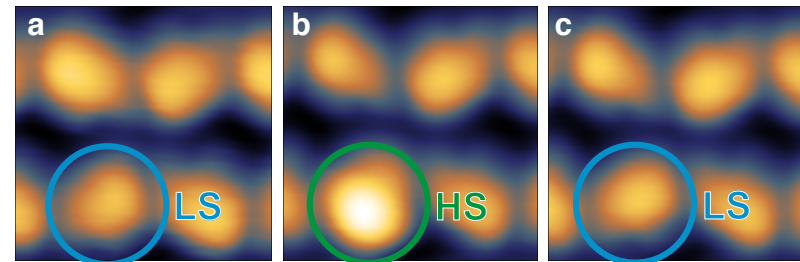

d

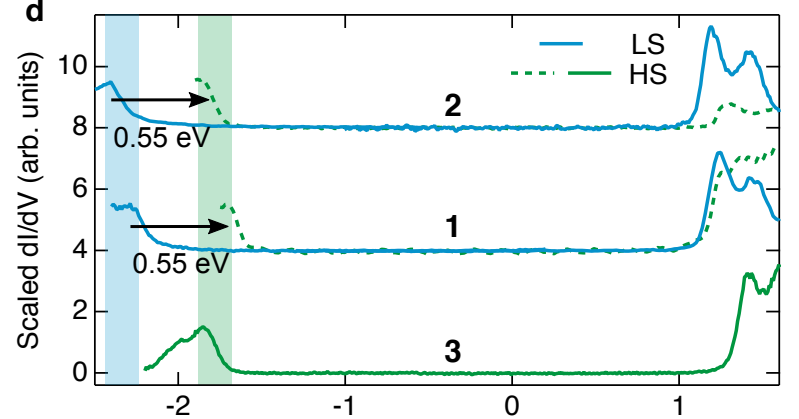

Sample voltage $(\mathrm{V})$

e

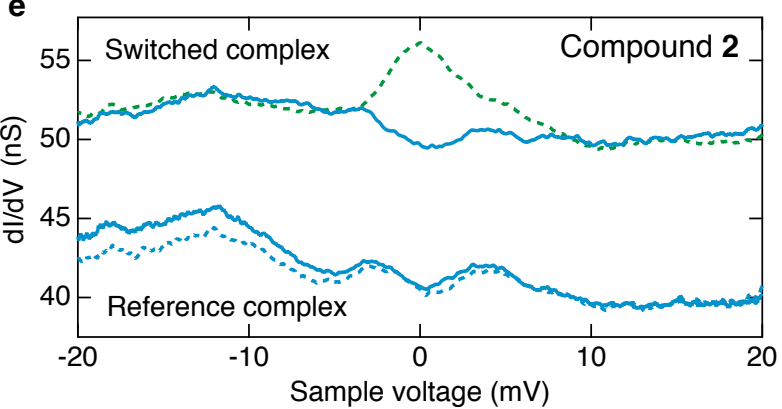

FIG. 4. Reversible coordination-induced spin-state switching of complexes 1-3 on $\mathbf{A g}(\mathbf{1 1 1})$. a - c Sequence of topographs ( $1 \mathrm{~V}, 10 \mathrm{pA}, 3.3 \mathrm{~nm}$ wide) illustrating reversible switching of 2 on $\operatorname{Ag}$ (111) from LS to HS and back to LS. To induce the transition from LS to HS (HS to LS), the sample voltage $V$ was increased (decreased) from 1.0 to $2.4 \mathrm{~V}$ at a current of $I \approx 100 \mathrm{pA}(-1.8 \mathrm{~V}$ at $I \approx 10 \mathrm{pA})$. The switching leads to a large change of the apparent height of the molecule $(130 \mathrm{pm})$. Colored circles indicate the manipulated molecule. d, $d I / d V$ spectra of $\mathbf{2}$ and $\mathbf{1}$ on $\mathrm{Ag}(111)$ displaying an upward shift of the HOMO as a molecule is switched from the pristine LS (blue curves) to the HS states (green curves). For 3 on $\operatorname{Ag}(111)$, only the HS state (green curve) was experimentally accessible. Note that the amplitude of different spectra may not be compared because they have been arbitrarily adjusted for clarity. For the $d I / d V$ spectra acquired on LS complexes, two sets of measurements where required at different tip-sample heights (100-150 pm height difference) because of the low conductance of the HOMO resonance compared to that of the LUMO. e $d I / d V$ spectra over a narrower voltage range acquired before (dashed curves) and after (solid curves) switching a single complex of $\mathbf{2}$ from HS to LS. Changes in the tip electronic structure are excluded in this voltage range, as evidenced from the similarity of the spectra acquired on a non-switched neighboring complex in the pristine state are shown before and after the manipulation. The feedback loop was opened at a set point of $1 \mathrm{nA}$ and $20 \mathrm{mV}$. For clarity, the green curve is shifted by $+1 \mathrm{nS}$, while the spectra acquired on the reference complex are shifted by $-10 \mathrm{nS}$. its HOMO. For completeness, we note that switching attempts of $\mathbf{3}$ with STM were unsuccessful, as expected from the data acquired in solution (SI section I).

Differential-conductance spectra close to the Fermi level provide independent evidence for spin switching. While the $d I / d V$ spectra of complexes $\mathbf{2}$ in the LS pristine state are essentially featureless (solid blue curves in Fig. 4e), switching of the complex to the HS state leads to the appearance of a peak at the Fermi level (dashed green curve in Fig. 4e). We exclude that this peak arises from a molecular orbital because its width $(\approx 4 \mathrm{mV})$ is significantly smaller than that of the HOMO $(\approx 200 \mathrm{mV})$. Instead, we attribute this peak to a Kondo resonance, which originates from the interaction of a localized spin with conduction electrons. Fitting of the differentialconductance spectrum using a Frota lineshape yields a Kondo temperature of approximately $50 \mathrm{~K}$ (SI section VI). In combination with the data presented above, the presence/absence of a Kondo resonance in the $d I / d V$ spectra is a direct evidence of switching between the HS and LS state. A similar readout of the spin state has been previously used for spin-crossover complexes ${ }^{6,7}$.

In conclusion, we proposed and demonstrated a novel concept for single molecule spin switches that are reversibly operated on a metal surface. Magnetic bistability is achieved by an intramolecular feedback between coordination and spin state in a ligand strapped $\mathrm{Ni}$ porphyrin. In addition, we evidence that the electronic and magnetic properties of the $\mathrm{Ni}$ center are not affected by the adsorption on $\operatorname{Ag}(111)$ and that the molecules can individually and reversibly undergo coordination-induced spin-state switching. Each of these states is found to be stable for days at $4 \mathrm{~K}$. These results hold great promise for molecular spintronics, in particular to control the spin polarization developing at the interface between molecules and (magnetic) metal surfaces ${ }^{1,2}$. Moreover, the coordination or non-coordination of the axial ligand influence the accessibility of small gaseous molecules to the central metal ion ${ }^{15}$. This may turn out useful for controlling the catalytic activity of a surface.

\section{Methods}

\section{Sample preparation}

Samples for NEXAFS and STM measurements were prepared as follows. $\mathrm{Ag}(111)$ substrates were cleaned by repeated cycles of $\mathrm{Ar}^{+}$sputtering and annealing to $550{ }^{\circ} \mathrm{C}$. The molecules were deposited by sublimation from a Knudsen cell ( $T$ between 250 and $290^{\circ} \mathrm{C}$ ) onto $\operatorname{Ag}(111)$ surfaces held at ambient temperature. The samples were transferred to the respective measurement chambers without breaking the ultra-high vacuum. The samples referred to as powder consist of a small quantity of powder deposited onto a carbon tape. 


\section{NEXAFS}

The NEXAFS measurements shown in the manuscript were performed at the DEIMOS beamline ${ }^{27-29}$ of the synchrotron SOLEIL. Preliminary experiments had been made at the X-TREME beamline of the Swiss Light Source $^{30}$. X-ray absorption was measured via the so-called total electron yield mode under a field of $0.1 \mathrm{~T}$ with circularly polarized photons unless otherwise specified. For the spectra of molecules on $\mathrm{Ag}(111)$, the angle between the x-ray beam and the sample normal was $55^{\circ}$. Background was removed from the spectra as described in the SI section IV. The spectra were averaged over 8 or 16 single measurements low-pass filtered to remove high-frequency noise and their integral was then normalized to 1 .

\section{STM}

The measurements were carried out with a STM operating in ultrahigh vacuum at $\approx 4 \mathrm{~K}$. STM topographs were recorded at constant tunneling current. Differential conductance spectra were acquired using a lock-in amplifier (667.8 Hz modulation) with current feedback disabled. To resolve molecular orbitals (a Kondo resonance), a modulation amplitude of $15 \mathrm{mV}_{\mathrm{RMS}}\left(700 \mu \mathrm{V}_{\mathrm{RMS}}\right)$ was applied. The spectra are the average of 60 sweeps.

\section{References}

* rherges@oc.uni-kiel.de and gruber@physik.uni-kiel.de

[1] Djeghloul, F. et al. High Spin Polarization at Ferromagnetic Metal-organic Interfaces: A Generic Property. $J$. Phys. Chem. Lett. 7, 2310-2315 (2016).

[2] Gruber, M. et al. Spin-Dependent Hybridization between Molecule and Metal at Room Temperature through Interlayer Exchange Coupling. Nano Lett. 15, 7921-7926 (2015).

[3] Barraud, C. et al. Unravelling the role of the interface for spin injection into organic semiconductors. Nat. Phys. 6, 615-620 (2010).

[4] Cinchetti, M., Dediu, V. A. \& Hueso, L. E. Activating the molecular spinterface. Nat. Mater. 16, 507-515 (2017).

[5] Delprat, S. et al. Molecular spintronics: the role of spindependent hybridization. J. Phys. D 51, 473001 (2018).

[6] Gopakumar, T. G. et al. Electron-Induced Spin Crossover of Single Molecules in a Bilayer on Gold. Angew. Chem. Int. Ed. 51, 6262-6266 (2012).

[7] Miyamachi, T. et al. Robust spin crossover and memristance across a single molecule. Nat. Commun. 3, 938 (2012).

[8] Bairagi, K. et al. Molecular-scale dynamics of lightinduced spin cross-over in a two-dimensional layer. Nat. Commun. 7, 12212 (2016).

[9] Knaak, T. et al. Fragmentation and Distortion of Terpyridine-Based Spin-Crossover Complexes on $\mathrm{Au}(111)$. J. Phys. Chem. C 123, 4178-4185 (2019).

[10] Wäckerlin, C. et al. Controlling spins in adsorbed molecules by a chemical switch. Nat. Commun. 1, 61 (2010).

[11] Seufert, K., Auwärter, W. \& Barth, J. V. Discriminative Response of Surface-Confined Metalloporphyrin Molecules to Carbon and Nitrogen Monoxide. J. Am. Chem. Soc. 132, 18141-18146 (2010).

[12] Wäckerlin, C. et al. Ammonia Coordination Introducing a Magnetic Moment in an On-Surface Low-Spin Porphyrin. Angew. Chem. Int. Ed. 52, 4568-4571 (2013).

[13] Hermanns, C. F. et al. Huge magnetically coupled orbital moments of Co porphyrin molecules and their control by CO adsorption. Phys. Rev. B 88, 104420 (2013).

[14] Gopakumar, T. G., Tang, H., Morillo, J. \& Berndt, R. Transfer of $\mathrm{Cl}$ Ligands between Adsorbed Iron Tetraphenylporphyrin Molecules. J. Am. Chem. Soc. 134, 11844-11847 (2012).

[15] Sugiyasu, K., Ogi, S. \& Takeuchi, M. Strapped porphyrin-based polymeric systems. Polym. J. 46, 674681 (2014).

[16] Venkataramani, S. et al. Magnetic Bistability of Molecules in Homogeneous Solution at Room Temperature. Science 331, 445-448 (2011).

[17] Matino, F. et al. Single azopyridine-substituted porphyrin molecules for configurational and electronic switching. Chem. Commun. 46, 6780-6782 (2010).

[18] Momenteau, M. \& Reed, C. A. Synthetic Heme-Dioxygen Complexes. Chem. Rev. 94, 659-698 (1994).

[19] Gazeau, S., Pécaut, J. \& Marchon, J.-C. Nickel porphyrin nanotweezers. Chem Comm 1644-1645 (2001).

[20] Zhang, X. et al. Ultrafast Stimulated Emission and Structural Dynamics in Nickel Porphyrins. J. Phys. Chem. A 111, 11736-11742 (2007).

[21] Duval, H., Bulach, V., Fischer, J. \& Weiss, R. FourCoordinate, Low-Spin $(S=0)$ and Six-Coordinate, HighSpin $(S=1)$ Nickel(II) Complexes of Tetraphenylporphyrins with $\beta$-pyrrole Electron-Withdrawing Substituents: Porphyrin-Core Expansion and Conformation. Inorg. Chem. 38, 5495-5501 (1999).

[22] Jia, S.-L. et al. Axial coordination and conformational heterogeneity of nickel(ii) tetraphenylporphyrin complexes with nitrogenous bases. Inorg. Chem. 37, 4402-4412 (1998).

[23] Gutzeit, F. et al. Structure and Properties of a FiveCoordinate $\mathrm{Ni}(\mathrm{II})$ porphyrin. Inorg. Chem. 58, 1254212546 (2019).

[24] Krasnikov, S. A. et al. Electronic structure of Ni(II) porphyrins and phthalocyanine studied by soft X-ray absorption spectroscopy. Chem. Phys. 332, 318-324 (2007).

[25] Gottfried, J. M. Surface chemistry of porphyrins and phthalocyanines. Surf. Sci. Rep. 70, 259-379 (2015).

[26] Auwärter, W., Écija, D., Klappenberger, F. \& Barth, J. V. Porphyrins at interfaces. Nat. Chem. 7, 105-120 (2015).

[27] Ohresser, P. et al. DEIMOS: A beamline dedicated to dichroism measurements in the $350-2500 \mathrm{eV}$ energy range. Rev. Sci. Instrum. 85, 013106 (2014).

[28] Joly, L. et al. Fast continuous energy scan with dynamic coupling of the monochromator and undulator at the DEIMOS beamline. J. Synchrotron Radiat. 21, 502-506 (2014). 
[29] Kappler, J.-P. et al. Ultralow-temperature device dedicated to soft X-ray magnetic circular dichroism experiments. J. Synchrotron Radiat. 25, 1727-1735 (2018).

[30] Piamonteze, C. et al. X-Treme beamline at SLS: X-ray magnetic circular and linear dichroism at high field and low temperature. J. Synchrotron Radiat. 19, 661-674 (2012).

\section{Acknowledgments}

We are grateful to the SOLEIL staff for smoothly running the facility, to F. Leduc for technical assistance and acknowledge funding by the Deutsche Forschungsgemeinschaft (DFG) via the SFB 677. This project has received funding from the European Union's Horizon 2020 research and innovation program under grant agreement No. 766726. Experiments were performed on the DEIMOS beamline at SOLEIL Synchrotron, France (proposal numbers 20171015 and 20190691) and associated costs have been supported by the project CALIPSOplus under Grant Agreement 730872 from the EU Framework Programme for Research and Innovation HORIZON 2020. This work has benefited from a funding from LabEx PALM (ANR-10-LABX-0039-PALM). M.S. acknowledges funding from the European Union's Horizon 2020 research and innovation programme under the Marie Sklodowska-Curie grant agreement No 701647 and Swiss National Science Foundation (Grant No. 200021_165774/1).

\section{Author contributions}

F.G. and R.H. conceived and synthesized the molecules. M.G., S.R., T.J.T., S.J., F.D., A.S., J.G. and M.S. carried out the NEXAFS measurements. M.G. analyzed the corresponding data. F.G. and F.R. carried out the density functional theory calculations. A.K., M.G. and A.W. performed the STM measurements and analyzed the data. All authors discussed the data and their interpretation. M.G. wrote the manuscript with input from R.B., A.K., F.G. and R.H.

\section{Data availability}

The data that support the plots within this paper and other findings of this study are available from the corresponding author upon reasonable request.

\section{Additional information}

Supplementary information is available in the online version of the paper. Reprints and permission information is available online at www.nature.com/reprints.
Correspondence and requests for materials should be addressed to M.G. 

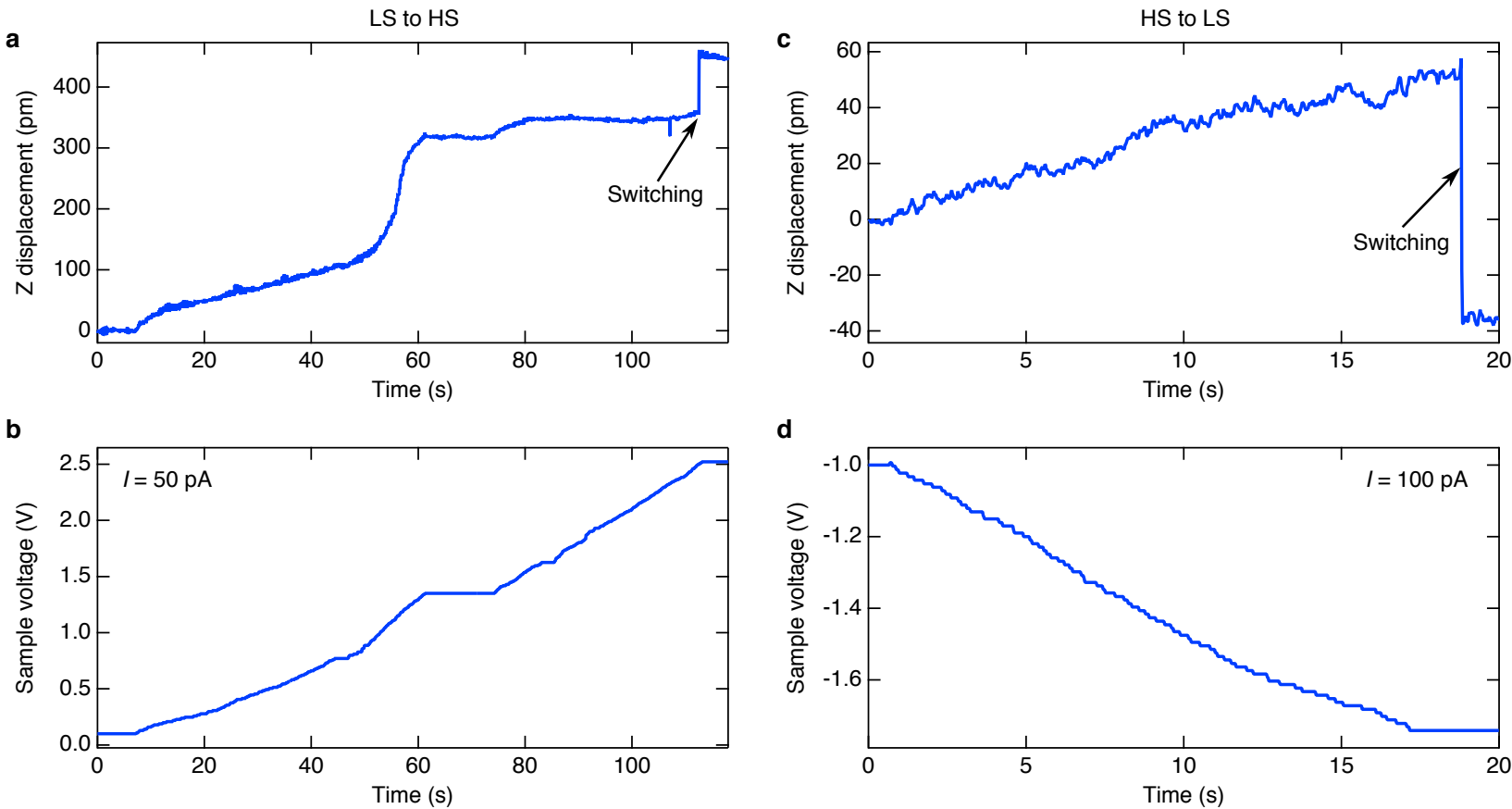

Extended Data Figure 1 - Description of the switching procedure of complex 2. Examples of switching procedures $\mathbf{a}-\mathbf{b}$ from the LS to the HS state and $\mathbf{c}-\mathbf{d}$ from the HS to the LS state. The tip is positioned over the center of the complex experiencing the switching, and the current feedback loop is active while the voltage is gradually varied as a function of time. While the switching from the LS to the HS state (HS to LS) occurs at a sample voltage of $2.5 \mathrm{~V}(-1.7 \mathrm{~V})$ for these examples, the voltage required to induce a transition varies from 2.1 to $2.7 \mathrm{~V}(-1.6$ to $-2.1 \mathrm{~V})$, and the large voltage may need to be applied for several seconds to several minutes for transitions from the LS to the HS state. The large spread in the threshold voltages may be due to the exact positioning of the tip and details of the molecule's environment. To increase the success rate of the switching from the LS to the HS state, we usually applied a sample voltage between 2.5 and $2.7 \mathrm{~V}$ with a current between $30 \mathrm{pA}$ and $1 \mathrm{nA}$ for approximately $5 \mathrm{~min}$. While this procedure is very effective, it is at the cost of selectivity

as neighboring molecules were usually switched as well. In contrast, HS to LS switching is relatively efficient. Sub-pA currents may be sufficient to induce the corresponding transition (see Extended Data Fig. 2). The arrows indicate the time at which the switching takes place. 

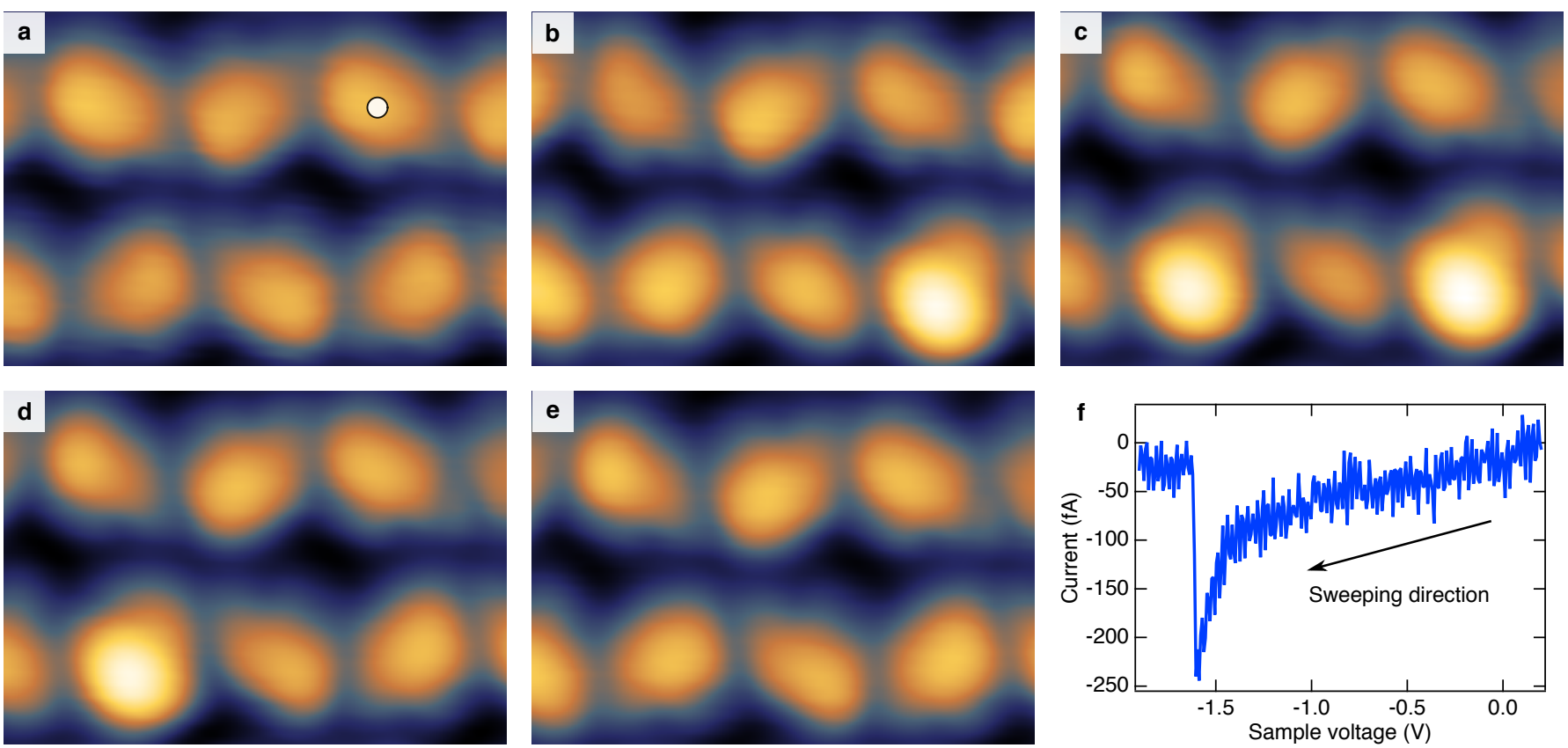

Extended Data Figure 2 - Switching sequence of two complexes. a-e Constant-height STM topographs (1 V, $10 \mathrm{pA}, 4.65 \mathrm{~nm}$ wide) describing the switching sequence of two complexes (compound 2) on $\mathrm{Ag}(111)$. In this example, a sample voltage of $2.7 \mathrm{~V}$ was applied for $30 \mathrm{~s}$ between the acquisition of topographs $\mathbf{a}$ and $\mathbf{b}$, and between topographs $\mathbf{b}$ and $\mathbf{c}$ (tunneling current of $30 \mathrm{pA}$ ) to induce transitions from the LS to the HS state of the lower left and lower right complexes.

The topographs $\mathbf{a}$ and $\mathbf{b}$ illustrate that the switching can be induced in neighboring molecules upon application of large voltages. Indeed, the tip was positioned on the top right molecule (white disk in a) while the lower right molecule is switched (lower right molecule in $\mathbf{b}$ ). f, $I-V$ curve recorded atop the lower right molecule in $\mathbf{c}$ leading to the switching from the HS to the LS state. Note that a current of $\approx 250 \mathrm{fA}$ was sufficient to induce the transition highlighting the efficiency of the backward switching process. 